\title{
Platelet-Derived Growth Factor-B Normalizes Micromorphology and Vessel Function in Vascular Endothelial Growth Factor-A-Induced Squamous Cell Carcinomas
}

\author{
Wiltrud Lederle, ${ }^{\star \dagger}$ Nina Linde, ${ }^{*}$ Julia Heusel, ${ }^{*}$ \\ Jessica Bzyl, ${ }^{\dagger}$ Eva C. Woenne, ${ }^{\ddagger}$ Stefan Zwick, ${ }^{\ddagger \S}$ \\ Mihaela Skobe, ${ }^{\text {"T }}$ Fabian Kiessling, ${ }^{\dagger}$ \\ Norbert E. Fusenig," and Margareta M. Mueller* \\ From the Tumor and Microenvironment Group (A101)," the \\ Department of Medical Physics in Radiology, ${ }^{\ddagger}$ and the Division of \\ Differentiation and Carcinogenesis (A080)," German Cancer \\ Research Center Heidelberg, Germany; the Department of \\ Experimental Molecular Imaging, ${ }^{\dagger}$ Medical Faculty, RWTH Aachen \\ University, Aachen, Germany; the Department of Diagnostic \\ Radiology, ${ }^{\S}$ Medical Physics, University Hospital, Albert Ludwigs \\ University, Freibung, Germany; and the Mount Sinai School of \\ Medicine, ${ }^{\text {Tा }}$ New York, New York
}

Vascular endothelial growth factor (VEGF), which is a key regulator of angiogenesis, often induces formation of immature vessels with increased permeability and reduced vessel functionality. Here, we demonstrate that de novo expression of murine (m)VEGF164 induces malignant and invasive tumor growth of HaCaT keratinocytes. However, the mVEGF-164-induced tumors are ulcerated with a disorganized epithelium that is interrupted by lacunae with limited basement membrane and endothelial cell coverage. Vessel maturation is strongly impaired. Tumor and vessel micromorphology are markedly improved by the combined expression of human platelet-derived growth factor (hPDGF)-B and mVEGF-164. Although tumor size and malignancy are comparable with either mVEGF-164 alone or combined human PDGF-B and mVEGF-164 expression, combined hPDGF-B and mVEGF-164 expression leads to a more solid and compact tumor tissue with a mature functional tumor vasculature and a higher microvessel density, as demonstrated histologically and by dynamic contrast-enhanced magnetic resonance imaging. Treatment of the hPDGF-B- and mVEGF-164-expressing tumors with imatinib mesylate to block PDGF-B signaling reverses this effect. In addition, tumor cell invasion of mVEGF-164 transfectants and mVEGF-164 plus hPDGF-B transfectants in vivo is associated with a marked induction of tumor-derived matrix metalloproteinase-1 and stromal matrix metalloproteinase-9 and $\mathbf{- 1 3}$, as was confirmed in three-dimensional organotypic co-cultures with fibroblasts in vitro. These data clearly demonstrate the need for a concerted action of different growth factors in the establishment of solid tumors with functional vasculature and emphasize the need for a multifactorial therapy. (Am J Pathol 2010, 176:981-994; DOI: 10.2353/ajpath.2010.080998)

Tumor angiogenesis is a complex multistep process and a crucial pre-requisite for tumor development, growth and progression. ${ }^{1}$ It includes the degradation of the extracellular matrix as well as the proliferation, migration, and differentiation of endothelial cells and is tightly controlled by stimulatory and inhibitory factors. ${ }^{2-4}$ One of the key angiogenic factors is vascular endothelial growth factor (VEGF), also known as vascular permeability factor, which plays an important role in physiological and pathological angiogenesis, eg, during wound healing ${ }^{5,6}$ and cancer. ${ }^{7,8}$ VEGF is up-regulated by different stimuli, eg, hypoxia ${ }^{9}$ or ras oncogene activation, ${ }^{10,11}$ and its overexpression in tumor cells enhances tumor growth ${ }^{12-14}$ and invasion. ${ }^{15-17}$ However, multiple studies have demonstrated that VEGF-induced blood vessels often show increased permeability, resulting in hemorrhages and reduced vessel functionality. ${ }^{18}$ These observations indicate that additional growth factors cooperate with VEGF dur-

Supported by the European Union FP6 Cancer Degradome and grants from the Deutsche Forschungsgemeinschaft (SFB-TR23 (project Z1) and SPP1190 Project MU1830/3-1).

Accepted for publication October 20, 2009.

Supplemental material for this article can be found on http://ajp. amjpathol.org.

Address reprint requests to P.D. Dr. Margareta M. Mueller, Tumor and Microenvironment (A101), German Cancer Research Center, INF 280, 69120 Heidelberg, Germany. E-mail: Ma.Mueller@dkfz.de. 
ing normal and pathological angiogenesis to establish mature, functional blood vessels with an intact basement membrane that can promote tumor growth by supplying the necessary nutrients to the tumor cell.

The platelet-derived growth factor (PDGF) isoform PDGF-B critically contributes to vessel stabilization by recruiting pericytes and smooth muscle cells. ${ }^{19,20}$ PDGF-B and PDGFR- $\beta$ knockout mice failed to sufficiently recruit pericytes to newly formed blood vessels, leading to severe perturbations in blood vessel stabilization and maturation. ${ }^{21,22}$ Lack of PDGF-B expression in endothelial cells resulted in the loss of pericytes around blood vessels and in vessel abnormalities. ${ }^{23}$ Besides its functions in physiological angiogenesis, PDGF-B is upregulated by many tumor cells, ${ }^{24,25}$ where it can act as an autocrine growth factor. Yet it also plays a crucial role in the establishment of functional vessels in the tumor. ${ }^{26,27}$ Tumor-inducing paracrine functions of PDGF-B have been demonstrated in our HaCaT skin carcinogenesis model. Transfection of the nontumorigenic keratinocyte cell line HaCaT with human (h)PDGF-B resulted in benign tumor growth that was mediated by initial stromal activation and subsequent stromal and vascular maturation. ${ }^{28,29}$ In this context, we observed an early PDGF-Bmediated up-regulation of stromal VEGF that indicated a crucial role of VEGF in the induction of tumor growth.

To further determine the specific role of VEGF in tumor growth, we stably transfected nontumorigenic $\mathrm{HaCaT}$ cells with murine (m)VEGF-A-164 and assessed tumor growth after s.c. injection and as surface transplants in nude mice. The combined effects of hPDGF-B and mVEGF-164 were analyzed using 1:1 mixed populations of hPDGF-B and mVEGF-164-transfected HaCaT cells in the s.c. tumor model and in the matrix inserted surface transplantation assay.

We demonstrate that de novo expression of mVEGF164 in the HaCaT cells induces malignant and invasive tumor growth. However, the mVEGF-164 transfectant tumors were ulcerated with a disorganized epithelium and interrupted by lacunae like structures with only limited basement membrane and endothelial cell coverage. In addition, blood vessel maturation was dramatically impaired. Tumor epithelial and vessel structures were markedly improved by the coexpression of hPDGF-B and mVEGF-164 in the 1:1 mixed cell populations, leading to a more compact tumor mass, an increased microvessel density and a mature functional tumor vasculature as demonstrated by histological analysis and by dynamic contrast-enhanced magnetic resonance imaging (DCE-MRI). The improved phenotype was reverted by blocking PDGF receptor (PDGFR) signaling with imatinib mesylate. These data clearly demonstrate that growth factors need to act in concert to establish solid tumor growth with a functional tumor vasculature.

\section{Materials and Methods}

\section{Cell Culture}

Immortal HaCaT keratinocytes (of human origin) were cultured as described previously. ${ }^{30} \mathrm{HaCaT}$ hPDGF-B transfectants resulted from transfection of the nontumorigenic HaCaT cells with a hPDGF-B expression vector (pcDNA1-PDGF-B) as described previously. ${ }^{28,29} \mathrm{HaCaT}$ mVEGF-164 transfectants and control transfectants were obtained by transfection of the parental $\mathrm{HaCaT}$ cells with the pcDNA1 expression vector containing the cDNA for mVEGF-A-164 or with pcDNA1, respectively, as described previously. ${ }^{17}$ The transfectants were maintained in the presence of $400 \mu \mathrm{g} / \mathrm{ml} \mathrm{G} 418$ (PAA, Cölbe, Germany).

Human dermal fibroblasts were derived from explant cultures of upper human dermis and cultivated in Dulbecco's modified Eagle's medium/10\% FCS (Biochrom, Berlin, Germany).

Cells were routinely tested for mycoplasma contamination as previously described ${ }^{31}$ and always found to be negative.

\section{Tumorigenicity Assay in Vivo: s.c. Injection}

For histological analyses, $5 \times 10^{6}$ cells in $100 \mu$ l were injected s.c. into both flanks of 5- to 6-week-old nude mice (four mice per group). Combined effects of mVEGF164 and hPDGF-B were analyzed by injecting mixed populations of HaCaT mVEGF-164 and HaCaT hPDGF-B transfectants in a ratio of 1:1 $\left(2.5 \times 10^{6}\right.$ cells each; mVEGF-164 plus hPDGF-B mixed tumors). Tumor size was measured weekly, and the tumor volume was calculated using the formula $\left(w^{2} \times I\right) \times 1 / 6 \pi$, where $w$ (width) represents the smallest and / (length) the largest diameter of the tumor. Tumors were removed $6,11,12,13$, and 14 weeks after injection.

For MRI analyses, $5 \times 10^{6}$ cells in $100 \mu$ l were injected into the right hind limb of nude mice (four animals per group). Tumor size was assessed as described above.

\section{Tumorigenicity Assay in Vivo: Surface Transplantation Assay}

Cells were transplanted onto the dorsal muscle fascia of 5- to 6-week-old nude mice (Swiss/c nu/nu) as organotypic cultures growing on a collagen type 1 gels as described previously. ${ }^{32,33}$ For the analysis of combined effects of hPDGF-B and mVEGF-164, HaCaT hPDGF-Band HaCaT mVEGF-164-transfected cells were transplanted in a ratio of $1: 1\left(1 \times 10^{5}\right.$ cells each). One to 6 weeks after transplantation animals were sacrificed, and transplants were removed, dissected, embedded in Tissue-Tek (Miles Laboratories, Elkhart, IN), frozen in liquid nitrogen, and processed for cryosectioning.

\section{Blockade of PDGFR Signaling in Vivo}

PDGFR signaling was blocked with imatinib mesylate ${ }^{34,35}$ (Glivec; Novartis Pharma, Basel, Switzerland) in nude mice either transplanted with a 1:1 mixture of $\mathrm{HaCaT}$ mVEGF-164 and HaCaT hPDGF-B transfectants or with HaCaT mVEGF-164 transfectants alone. Imatinib mesylate was given by oral gavage in $100 \mu \mathrm{l}$ of saline solution at a dose of $150 \mathrm{mg} \times \mathrm{kg}^{-1}$ body weight $\times$ day $^{-1}$ starting at week 3 after transplantation. Treatment was continued for 3 weeks until week 6 posttransplantation. 


\section{Tumorigenicity Assay in Vitro: Organotypic Co-Cultures}

Dermal equivalents were prepared with native type I rat collagen by mixing eight volumes of ice-cold collagen solution ( $4 \mathrm{mg} / \mathrm{ml}$ in $0.1 \%$ acetic acid) with 1 volume of $10 \times$ HBSS, followed by neutralization with $2 \mathrm{~mol} / \mathrm{L} \mathrm{NaOH}$. One volume of FCS with human dermal fibroblasts was added and mixed thoroughly, resulting in a final collagen concentration of $3 \mathrm{mg} / \mathrm{ml}$. Of this mixture, $2.5 \mathrm{ml}$ each were poured into polycarbonate membrane filter inserts (Falcon number 3501; BD Biosciences, San Jose, CA), placed in special deep 6-well trays (BD Biosciences), allowed to solidify at $37^{\circ} \mathrm{C}$, and equilibrated with Dulbecco's modified Eagle's medium, 10\% FCS, and $50 \mu \mathrm{g} / \mathrm{ml}$ L-ascorbic acid (Sigma-Aldrich, Taufkirchen, Germany). Tumor cells $\left(8.5 \times 10^{5}\right.$ of HaCaT control, mVEGF-164 or hPDGF-B transfectants as well as a 1:1 mixture of mVEGF-164 and hPDGF-B transfectants, $4.25 \times 10^{5}$ cells each) were seeded on top of the collagen matrix. After submersed incubation overnight, the cultures were raised to the air-medium interface. For 4 weeks, two cultures per week were harvested and processed for cryostat sectioning. Data shown are representatives of two independent experiments with two replicas each.

\section{Antibodies and cDNAs for in Situ Hybridization}

Primary antibodies used: rat monoclonal antibody against mouse CD31 (BD Pharmingen, Heidelberg, Germany), guinea pig pan-keratin anti-serum (Progen, Heidelberg, Germany), rabbit polyclonal antibody against Ki-67 (Acris, Bad Nauheim, Germany), rabbit polyclonal collagen type IV antibody (Novotec, Lyon, France), rabbit polyclonal collagen type VII antibody (a gift from Dr. L. Bruckner-Tuderman, Department of Dermatology, University Medical Center Freiburg, Freiburg, Germany), biotinylated mouse monoclonal antibody against $\alpha$-smooth muscle actin (SMA) (Progen, Heidelberg, Germany), and goat affinity-purified antibody against mVEGF (R\&D Systems, Wiesbaden, Germany).

Secondary antibodies were obtained from Dianova (Hamburg, Germany).

The following probes were used for in situ hybridization: 1800-bp fragment of human matrix metalloproteinase MMP-1 cDNA, 363-bp fragment of mouse MMP-13 cDNA (all gifts from Dr. P. Angel, German Cancer Research Center, Heidelberg, Germany), and 220-bp fragment of mouse MMP-9 cDNA (Novus, San Diego, CA).

\section{Indirect Immunofluorescence}

Frozen sections were fixed for 5 minutes in $80 \%$ methanol at $4^{\circ} \mathrm{C}$, followed by 2 minutes in acetone at $-20^{\circ} \mathrm{C}$ and rehydrated in PBS. The staining procedure was performed as described previously. ${ }^{36}$ For staining of cell nuclei sections were incubated with $20 \mu \mathrm{g} / \mathrm{ml}$ Hoechst 33258/bisbenzimide together with the secondary antibody (Sigma-Aldrich). Stained sections were examined and photographed with the Leica DM RBE microscope (Leica, Bensheim, Germany) fitted with epifluorescence.

\section{In Situ Hybridization}

In situ hybridization was performed essentially as described previously. ${ }^{37}$ For nonradioactive in situ hybridization, digoxigenin signals were detected by anti-digoxigenin-AP (Roche, Mannheim, Germany) and alkaline phosphatase reaction with nitroblue tetrazolium/5-bromo-4-chloro-3-indolyl phosphate substrate (Promega, Mannheim, Germany). After in situ hybridization with MMP-1, MMP-9, or MMP-13 probes, counterstaining by indirect immunfluorescence was performed with antisera against pan-keratin and collagen type IV. Stained sections were examined and photographed with the Leica DM RBE microscope (Leica) fitted with epifluorescence. For better visualization of DIG in situ signals, colors were reassigned using the analySIS software (Soft Imaging Systems, Münster, Germany).

\section{Enzyme-Linked Immunosorbent Assay}

Secreted mVEGF-164 protein in conditioned media was measured by enzyme-linked immunosorbent assay using the Quantikine Immunoassay Kit from R\&D Systems (Wiesbaden, Germany) according to the manufacturer's instructions. Samples were tested in duplicate. The data shown are mean values of three independent experiments.

\section{Morphometric Analysis}

Quantification of compact epithelial tumor mass in s.c. tumors was performed by calculating the ratio of keratin stained area to the total epithelial area (sections of four animals per group). Blood vessel density in s.c. tumors was quantified by determining the ratio of CD31-positive area to the total area (for four animals each). The degree of vessel maturation in surface transplants was determined by calculating the ratio of SMA-stained area to CD31-stained area (sections of four animals per week and group).

\section{Noninvasive Characterization of Tumor Vascularization by DCE-MRI}

For noninvasive imaging and catheterization, mice were anesthetized by inhalation of a mixture of isoflurane (1.5\%), $\mathrm{N}_{2} \mathrm{O}(35 \%)$, and $\mathrm{O}_{2}(60 \%)$. The tail vein was catheterized using a 30-gauge needle connected to a $10-\mathrm{cm}$ long PE 10 polyethylene catheter (Portex, Medic-Eschmann, Germany) filled with $10 \mu$ of $0.9 \% \mathrm{NaCl}$. Successful catheterization was controlled by blood reflux into the catheter and by injection of $30 \mu \mathrm{l}$ of $0.9 \% \mathrm{NaCl}$. After each examination, the needle was removed from the tail vein.

Relative blood volume and vessel permeability were determined by DCE-MRI on a clinical 1.5 T whole-body MRI system (Siemens Magnetom Vision, Erlangen, Germany), using a custom-made active radiofrequency coil. Animal and tumor morphology were assessed using a high resolved T1w 2D spinecho sequence (TR (relaxation 
time) $600 \mathrm{~ms}$; TE (echo time) $17 \mathrm{~ms}$; FOV (field of view) $50 \times 32 \mathrm{~mm}^{2}$; voxel size $0.1 \times 0.1 \times 1.0 \mathrm{~mm}^{3}$; averages 3) and a T2w turbo spinecho sequence (TR $1510 \mathrm{~ms}$; TE $59 \mathrm{~ms}$; FOV $50 \times 32 \mathrm{~mm}^{2}$; voxel size $0.4 \times 0.4 \times 1 \mathrm{~mm}^{3}$; averages 15), followed by DCE-MRI. DCE-MRI was performed using a T1w saturation recovery turbo FLASH sequence (TR $13 \mathrm{~ms}$, TE 5,3 ms, TI (inversion time) 300 $\mathrm{ms}$, Flip angle $12^{\circ}$, averages 4 , FOV $60 \times 22.5 \mathrm{~mm}^{2}$, voxel size $0.5 \times 0.5 \times 2.0 \mathrm{~mm}^{3}$, time per scan $7.5 \mathrm{~s}$, number of scans 120). Gadomer $(0.05 \mathrm{mmol} / \mathrm{kg}$ diluted in $0.9 \% \mathrm{NaCl}$ to a total volume of $100 \mu \mathrm{l} /$ mouse; BayerSchering-Pharma, Berlin, Germany) was administered as contrast agent via tail vein injection. Postprocessing was done according to the two compartment model of Brix $^{38}$ using DynaLab (Mevis, Bremen, Germany). The two parameters, amplitude $(A)$ and exchange rate constant $\left(k_{\mathrm{ep}}\right)$, were determined for each pixel of the MR images. The amplitude $A$ is proportional to the degree of signal enhancement and used as a parameter of the relative tissue blood volume. The exchange rate constant $k_{\mathrm{ep}}$ describes the exchange of contrast agent between extravascular-extracellular space and blood and depends on perfusion and vessel permeability.

\section{Statistics}

A non parametric Mann-Whitney test was used for data analysis, since analysis of the collected data indicated no normal distribution. A value of $P<0.05$ was considered statistically significant.

\section{Results}

\section{De Novo Expression of mVEGF-164 Leads to Malignant Tumor Growth}

To analyze the effects of VEGF-A on tumor growth in the $\mathrm{HaCaT}$ skin carcinogenesis model, nontumorigenic HaCaT keratinocytes were transfected to express mVEGF164. mVEGF-164 expression was confirmed by RT-PCR with mouse-specific primers (data not shown) and by enzyme-linked immunosorbent assay. A production of 800 to $900 \mathrm{pg} / \mathrm{ml} \mathrm{mVEGF}$ per $10^{7}$ cells was measured in 48 hours conditioned media. All analyzed $\mathrm{HaCaT}$ cell lines (parental $\mathrm{HaCaT}$ cells, control transfectants, $\mathrm{HaCaT}$ mVEGF-164 transfectants, HaCaT hPDGF-B transfectants) showed an equal basal expression level of human VEGF, both at the mRNA and the protein level. In accordance with previous results, ${ }^{39}$ the basal human VEGF expression in these non-ras-transfected $\mathrm{HaCaT}$ cells was not sufficient to promote invasive tumor growth. No expression of the VEGF receptors, neither VEGFR-1 nor VEGFR-2, was detected in HaCaT cell monolayer cultures. In agreement with this, $\mathrm{HaCaT}$ cell proliferation was not influenced by exogenously added human VEGF-A or by the overexpression of mVEGF-164 (data not shown).

To assess the effect of mVEGF-164 in vivo, HaCaT mVEGF-164 and control transfectants were s.c. injected into nude mice. As expected, the HaCaT control transfectants were nontumorigenic. Only one of eight injec- tions gave rise to a small tumor nodule. Histological analysis confirmed a benign avascular cornified cyst (Figure 1A). In contrast, the mVEGF-164 transfectants induced tumors with sizes up to $650 \mathrm{~mm}^{3}$ after 12 weeks. These tumors were ulcerated and red colored, indicating an extensive vascularization (Figure 1B). On dissection, large blood vessels became apparent leading from the mouse back skin to the tumor mass. Histological analysis of the mVEGF-164 transfectant tumors revealed a vascularized, invasive tumor tissue. However, the tumor epithelium appeared disorganized with keratinized central parts and a heterogeneous vital tumor area disrupted by large lacunae-like structures (Figure 1B).

\section{Coexpression of mVEGF-164 and hPDGF-B Leads to Similar Tumor Growth but Improves Tumor Organization}

The irregular shape and aberrant morphology of the vessels in the mVEGF-164-induced tumors indicated a VEGF-mediated impairment in vessel maturation and function. Since PDGF-B is an important mediator of vessel maturation, ${ }^{20,40,41}$ combined effects of mVEGF-164 and $h P D G F-B$ were assessed by injecting a 1:1 mixture of HaCaT mVEGF-164 and HaCaT hPDGF-B transfectants into nude mice (mVEGF-164 plus hPDGF-B mixed tumors).

In contrast to small benign cysts that developed after a latency period of 6 to 8 weeks in response to s.c. injection of HaCaT hPDGF-B cells alone, ${ }^{28,29}$ coinjection of mVEGF-164 transfectants plus hPDGF-B transfectants resulted in tumors with similar size as the mVEGF-164 transfectant tumors (400 to $>600 \mathrm{~mm}^{3}$ within 12 weeks). Histological staining revealed again an invasive and wellvascularized tumor tissue with fewer and smaller lacunae and a more compact structure of the tumor mass (Figure 1C). This impression was confirmed by costaining of CD31-positive endothelial cells and keratin expressing tumor cells. Whereas mVEGF-164 transfectant tumors exhibited irregularly shaped blood vessels and large lacunae that were only partially lined by endothelial cells (Figure 2A), tumor structure was much more compact in the mVEGF-164 plus hPDGF-B mixed tumors. Tumor infiltrating blood vessels were generally smaller in size (Figure 2A). Quantification revealed a higher ratio of keratin stained area to total tumor area in the mVEGF-164 plus hPDGF-B mixed tumors, thus confirming an increase in compact epithelial tumor mass (Figure 2B).

To further analyze whether hPDGF-B also had an influence on vascular maturation, we analyzed the association of SMA-positive pericytes with the newly formed vessels in the tumor tissue. In s.c. tumors of the mVEGF-164 transfectants, blood vessels infiltrating the tumor tissue were free of SMA-positive pericytes. SMA covered blood vessels were restricted to the stromal rim of the tumor (Figure 2C, two left panels, arrows designate SMA-positive vessels, arrowheads mark endothelial free areas of the lacunae). In contrast, tumor-infiltrating vessels in the mVEGF-164 plus hPDGF-B mixed tumors were partially SMA positive (Figure 2C, two right panels, arrows mark SMA-positive vessels), 

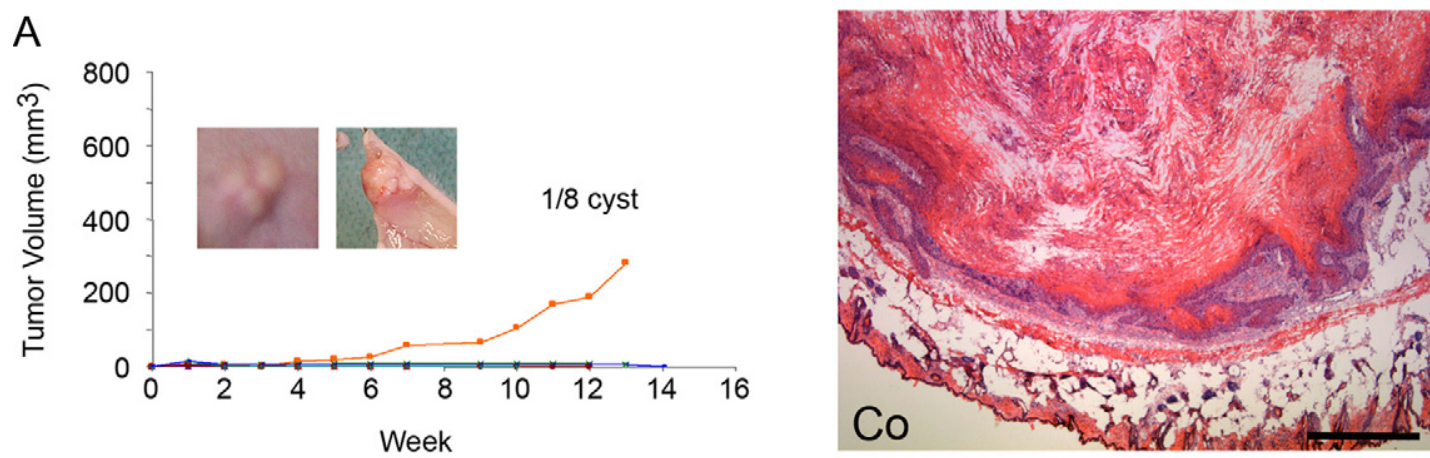

B
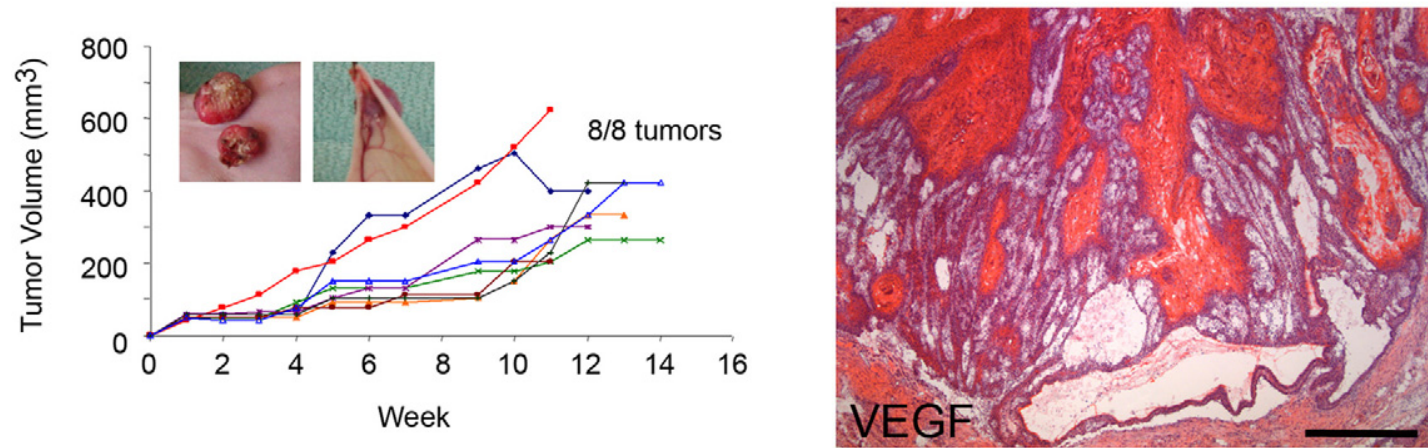

C
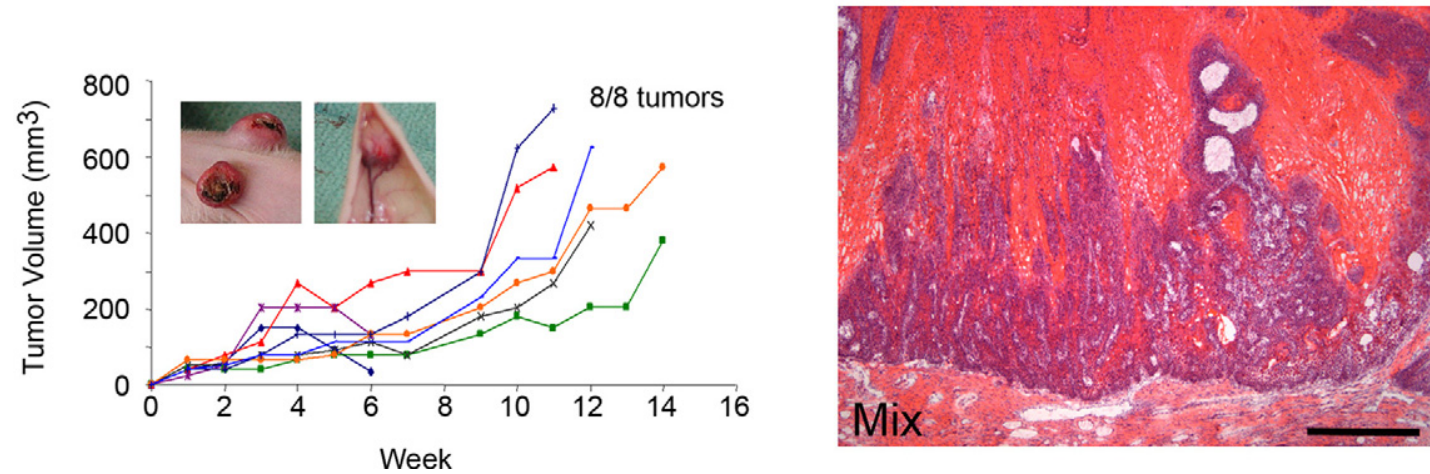

Figure 1. Malignant conversion of HaCaT cells by de novo expression of murine VEGF-164 and the coexpression of mVEGF-164 and hPDGF-B. A-C: Tumor growth curves (left panels) and H\&E staining (right panels) of s.c. tumors of HaCaT control transfectants (Co, A), HaCaT mVEGF-164 transfectants (VEGF, B), and a 1:1 mixture of HaCaT mVEGF-164 and hPDGF-B transfectants (Mix, C); the respective cells were injected into both flanks of four animals each. Scale bar, $500 \mu \mathrm{m}$.

suggesting an improved vessel maturation by the coexpression of mVEGF-164 and hPDGF-B.

Immunostaining for murine VEGF-A confirmed a marked VEGF expression in the mVEGF-164 transfectant tumors as well as in the mVEGF-164 plus hPDGF-B mixed tumors and demonstrated the enrichment of VEGF around blood vessels (Supplemental Figure S1, see http://ajp. amjpathol.org).

\section{Kinetics of Angiogenesis and Epithelial Growth in Surface Transplants}

To determine the specific effects of mVEGF-164 or mVEGF-164 plus hPDGF-B on the kinetics of angiogenesis, vessel maturation and epithelial growth, mVEGF164 transfectants, control transfectants as well as a 1:1 mixture of mVEGF-164 and hPDGF-B transfectants were transplanted as preformed epithelia on a collagen type I gel onto the dorsal muscle fascia of nude mice.

In accordance with previous observations, control transfectants displayed typical features of nontumorigenic keratinocytes. ${ }^{29}$ As demonstrated by immunostaining for CD31 and keratin, the epithelium increased during the observation period and remained separated from the stroma throughout the whole observation period of 6 weeks (Figure 3A, upper panels, shown for weeks 2 and $6)$. Induction of angiogenesis was delayed and blood vessel density in the stroma remained low.

The angiogenic response was markedly accelerated in transplants of HaCaT mVEGF-164 transfectants compared with the nontumorigenic controls with a dense assembly of vascular structures reaching the epithelial border in week 2. However blood vessels at the epithelial border were small, tortuous and irregularly shaped (Fig- 
A

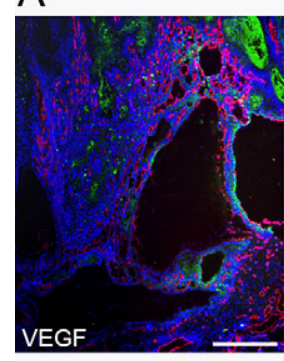

C

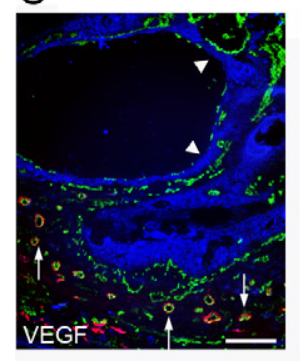

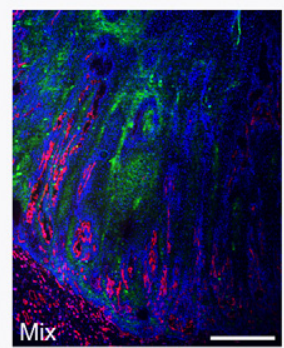

CD31/ Keratin/ Hoechst

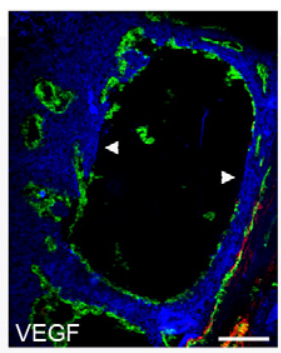

B
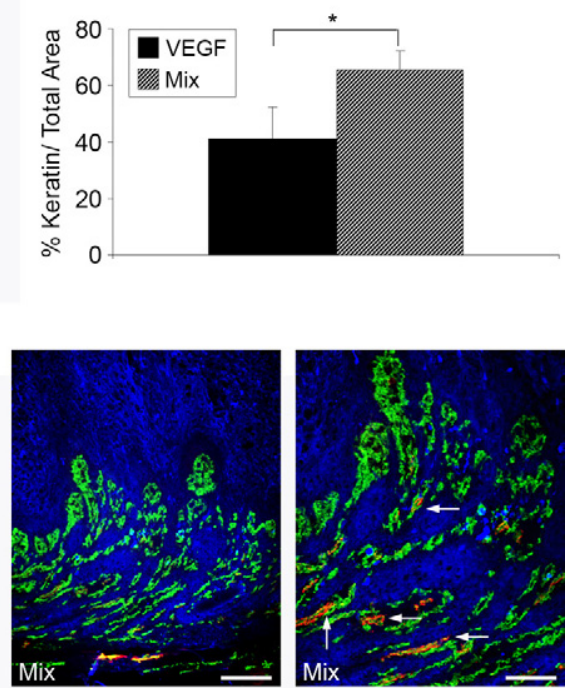

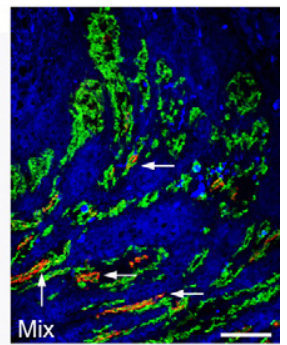

SMA CD31/ Keratin

Figure 2. Improved tissue morphology and vessel maturation by the coexpression of mVEGF164 and hPDGF-B. A: Immunostaining for CD31 (endothelial cells, red), pan-keratin (tumor tissue, green), and Hoechst (nuclei, blue) of cryosections from s.c. tumors of mVEGF-164 transfectants as well as mVEGF-164 plus hPDGF-B transfectants (Mix); bar, $500 \mu \mathrm{m}$. B: Quantification of keratin-stained area in relation to the total tumor area reveals an increase in compact epithelial tumor mass in the mVEGF-164 plus hPDGF-B mixed tumors (Mix). The bars represent the means $\pm \mathrm{SD} ;{ }^{*} P<0.05$. C: Immunostaining for SMA (pericytes, red), CD31 (endothelial cells, green), and pan-keratin (tumor tissue, blue) in s.c. tumors of mVEGF-164 transfectants (VEGF) and mVEGF-164 plus hPDGF-B transfectants (Mix) shows the presence of SMApositive vessels within the tumor tissue only in the mixed tumors (right panels). Arrows designate SMA-positive vessels, and arrowheads mark endothelial free areas in lacunae of mVEGF164 transfectant tumors. Scale bar, $200 \mu \mathrm{m}$ (first and third panel) and $100 \mu \mathrm{m}$ (second and fourth panel).

ure $3 \mathrm{~A}$, middle panels, left picture and quantification in 3B). At week 6 posttransplantation, an invasive, well vascularized tumor tissue with lacunae developed that strongly resembled the s.c. tumors (Figure 3A, middle panels, right picture and compare to Figure 2). In mVEGF-164 plus hPDGF-B mixed transplants, induction of angiogenesis occurred with similar accelerated kinetics as in transplants of the mVEGF-164 transfectants (Figure 3A lower panels and quantification in 3B). However, in week 2 the infiltrating blood vessels showed a more regular shape being directed toward the tumor epithelium (Figure 3A, lower panels, left picture and Sup-
A
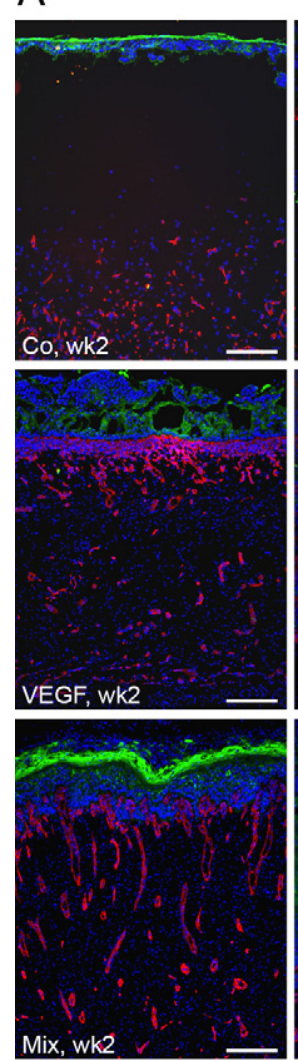

Mix, wk6

CD31/ Keratin/ Hoechst

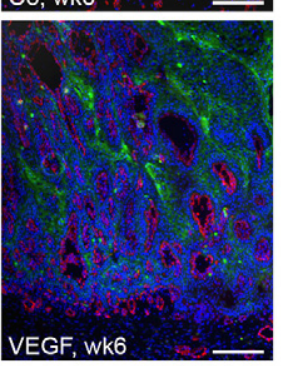

B

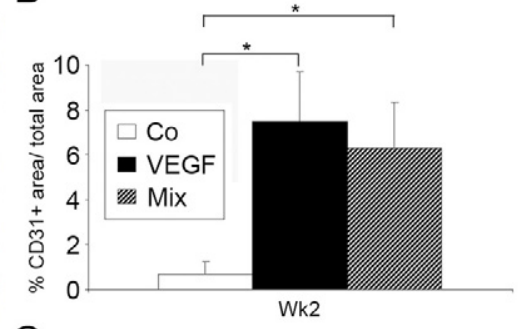

C
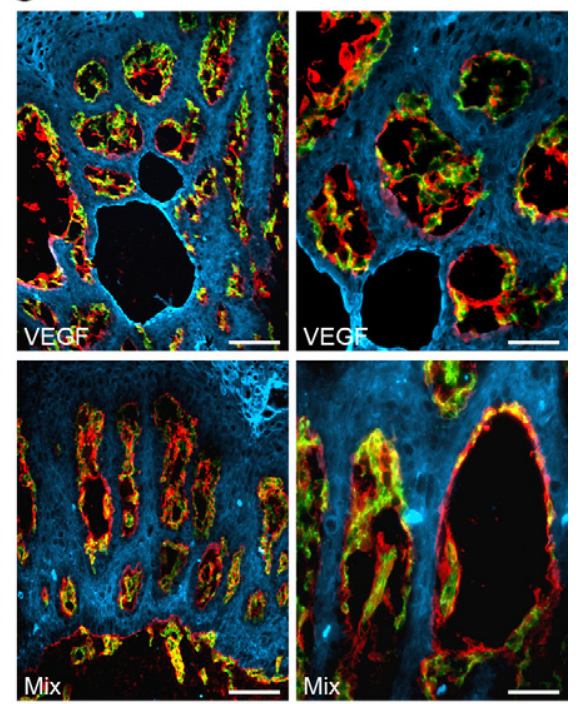

Col IVI CD31/ Keratin
Figure 3. Kinetics of epithelial growth and angiogenesis in the surface transplants. A: Indirect immunofluorescence of cryosections from 2 and 6-week-old transplants of control transfectants (Co), mVEGF-164 transfectants (VEGF), and mVEGF-164 plus hPDGF-B transfectants (Mix) with antibodies against CD31 (endothelial cells, red), pan-keratin (tumor tissue, green) and Hoechst (nuclei, blue); bar, $200 \mu \mathrm{m}$. mVEGF-164 and mVEGF-164 plus hPDGF-B accelerates angiogenesis and leads to invasive tumor growth B: Quantification of the ratio of CD31-stained area to the total area in surface transplants of week 2 confirms the accelerated angiogenesis of the mVEGF-164 and mVEGF-164 plus hPDGF-B mixed transplants. The bars represent the means $\pm \mathrm{SD} ;{ }^{*} P<0.05$. C: Immunofluorescent staining for CD31 (endothelial cells, green), collagen IV (vascular basement membrane, red), and pan-keratin (tumor cells, blue) in the tumor tissue of late mVEGF-164 (VEGF) and mVEGF164 plus hPDGF-B mixed transplants (Mix). Note the large lacunae devoid of endothelial cells and collagen IV (Col IV) in the tumor tissue of the mVEGF-164 transplants; Scale bar, $100 \mu \mathrm{m}$ (left panels) and $50 \mu \mathrm{m}$ (right panels). 
plemental Figure S2, see http://ajp.amjpathol.org). ${ }^{29}$ At week 6 posttransplantation, the tumor tissue was invasive, well vascularized, yet more compact than the tissue of the mVEGF-164 transfectant transplants (Figure 3A, lower panels, right picture). Invasive growth of the tumor cells in transplants of HaCaT mVEGF-164 transfectants and in the mVEGF-164 plus hPDGF-B mixed transplants was confirmed by the drastic reduction or the complete lack of staining for the basement membrane component collagen VII in contrast to the continuous staining in benign HaCaT hPDGF-B transfectant transplants (Supplemental Figure S2, see http://ajp.amjpathol.org).

The differences in epithelial structure in the HaCaT mVEGF-164 versus the mVEGF-164 plus hPDGF-B mixed transplants became even more obvious by costaining of CD31 and collagen IV in the tumor epithelium. In 6-weekold transplants of HaCaT mVEGF-164 transfectants, some lacunae were only partially lined by CD31-positive endothelial cells and/or collagen IV, and others showed no staining for either one of these markers (Figure 3C, upper panels). In contrast, the lacunae like structures were smaller and always positive for CD31 and collagen IV in the age-matched mVEGF-164 plus hPDGF-B mixed transplants (Figure 3C, lower panels).

\section{Improved Vessel Maturation and Epithelial Morphology in Transplants Coexpressing mVEGF-164 and hPDGF-B Is Reverted by PDGFR Blockade}

Vessel maturation in the surface transplants was assessed by immunostaining for SMA and CD31. No SMA positive vessels were observed in the upper part of HaCaT control transplants (Figure 4A). In surface transplants of the mVEGF-164 transfectants, SMA positive cells that were not associated with blood vessels, presumably myofibroblasts, were observed in the stroma already at week 2 and increased until week 6 posttransplantation (Figure 4B). At the same time the blood vessels in the upper stromal compartment and the tumor infiltrating vessels were predominantly SMA free (Figure 4B). The accumulation of myofibroblasts was even stronger in early transplants of mVEGF-164 plus hPDGF-B transfectants, however, we also detected an increased number of SMA positive blood vessels (Figure 4C). While the SMA positive myofibroblasts disappeared from the stroma, the number of SMA coated vessels further increased at week 6 posttransplantation and SMA-positive vessels were even detected in the tumor tissue (Figure 4C), thus confirming an improved vessel maturation as observed in the s.c. tumors.

To analyze whether the improved vessel maturation and epithelial structure of the mVEGF-164 plus hPDGF-B mixed transplants were indeed mediated by the expression of hPDGF-B in the tumors, we blocked PDGFR signaling by treatment with imatinib starting from week 3 until week 6 posttransplantation. While the recruitment of myofibroblasts was reduced after 3 weeks of imatinib treatment in surface transplants of HaCaT mVEGF-164 transfectants compared with age matched untreated controls (week 6 posttransplantation), PDGFR signaling blockade had no effect on the amount of SMA-associated vessels and the irregular structure of the epithelial tumor tissue (Figure 4, D and E, arrowheads mark endothelial free areas in the lacunae). In contrast, the number of SMA associated blood vessels was significantly decreased in the upper stroma of mVEGF-164 plus hPDGF-B mixed transplants, treated with imatinib for 3 weeks, indicating a strong impairment of vessel maturation after PDGFR signaling blockade (Figure 4F). Quantification of SMA-positive vascular structures revealed a significant decrease in the upper stromal part of mVEGF-164 plus hPDGF-B mixed transplants, treated with imatinib (Figure $4 \mathrm{H})$. Additionally, the treatment also reduced the association of SMA-positive pericytes with the blood vessels in the tumor tissue (Figure 4G, right panel, arrows), whereas large blood vessels in the lower stromal compartment were not affected, as obvious by their strong SMA coating (Figure $4 \mathrm{~F}$, right panel). Concomitant with the effect on vessel maturation, imatinib also had an effect on epithelial organization (Figure 4G). Large epithelial lacunae appeared in the tumor epithelium of mVEGF-164 plus hPDGF-B mixed transplants, treated with imatinib for 3 weeks, strongly resembling the epithelial structure of the HaCaT mVEGF-164 transplants and tumors (Figure 4E). These data clearly demonstrate that the normalizing effects on the tumor vessels and the epithelium were indeed mediated by hPDGF-B.

\section{Increased Microvessel Density and Decreased Vessel Permeability by the Coexpression of mVEGF-164 and hPDGF-B (DCE-MRI)}

The improved association of SMA-positive periyctes with blood vessels in mVEGF-164 plus hPDGF-B mixed tumors raised the question whether the PDGF-B coexpression also improved vessel function. To analyze vessel function, we injected $\mathrm{HaCaT}$ mVEGF-164 transfectants as well as a 1:1 mixture of HaCaT mVEGF-164 and HaCaT hPDGF-B transfectants into the right hind limb of nude mice and performed DCE-MRI 10 to 14 weeks later. The amplitude A, a parameter for the relative blood volume as well as the exchange rate constant kep, a marker for vessel permeability, were determined in the vascularized vital outer tumor area according to the pharmacokinetic two compartment model of Brix. The amplitude A was markedly increased in mVEGF-164 plus hPDGF-B mixed s.c. tumors compared with mVEGF-164 transfectant tumors, revealing an increased relative blood volume (Figure 5A). In agreement with this, histological quantification of vascularization on cryosections confirmed an increased vessel density in the vital area of mVEGF-164 plus hPDGF-B mixed S.c. tumors (Figure 5B). In contrast, the marker for blood vessel permeability kep was markedly lower than in HaCaT mVEGF-164 transfectant tumors (Figure 5C). This suggested an hPDGF-B-mediated decrease in vessel leakiness and an improved vessel function by the presence of more mature SMA-covered blood vessels. 
A

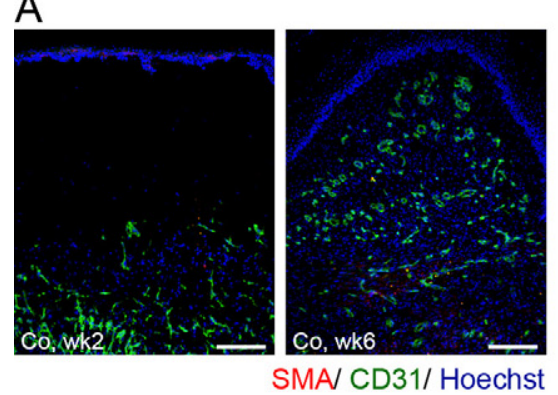

C

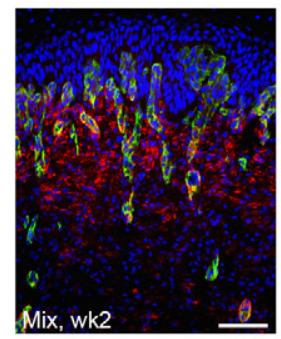

E

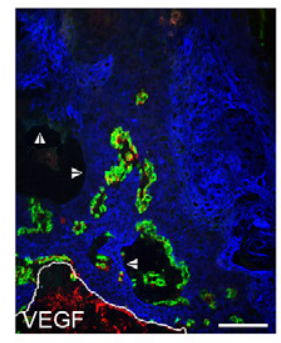

G
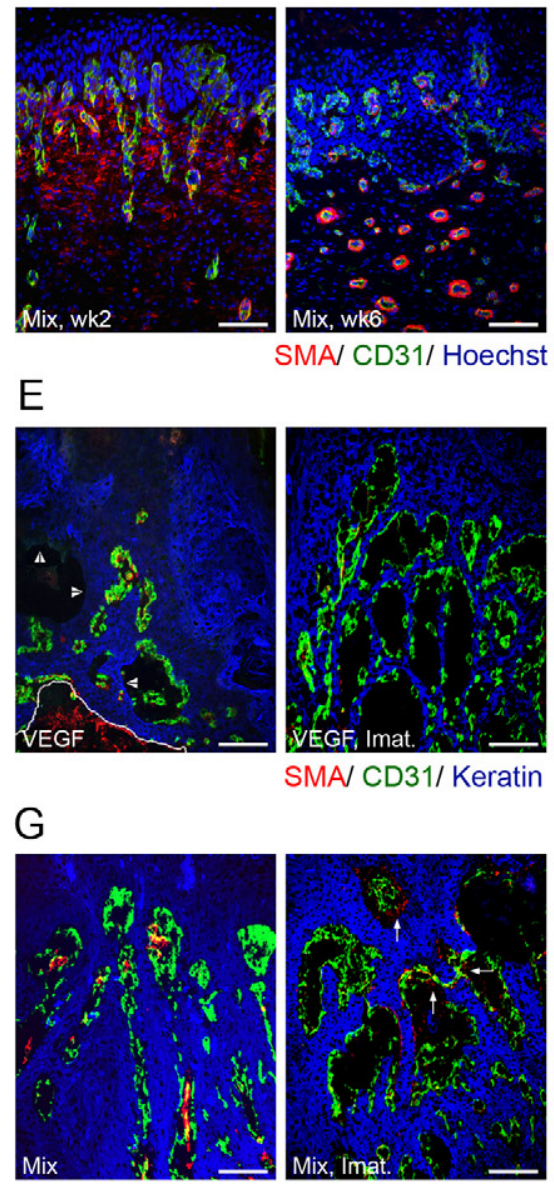

SMAV CD31/ Hoechst

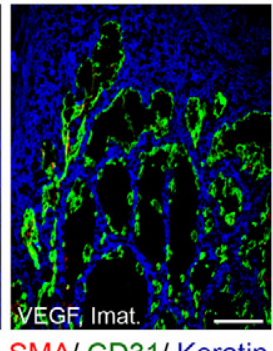

SMAV CD31/ Keratin
B

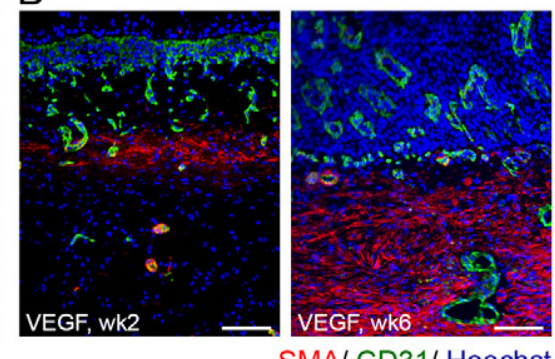

D

\section{$\mathrm{H}$}

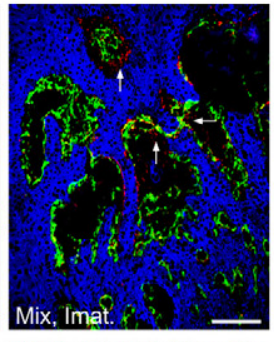

SMA CD31/ Keratin

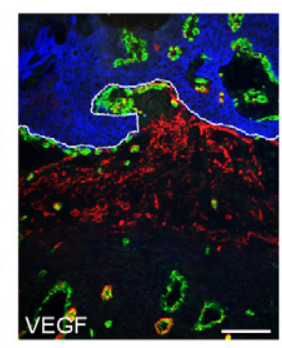

$\mathrm{F}$

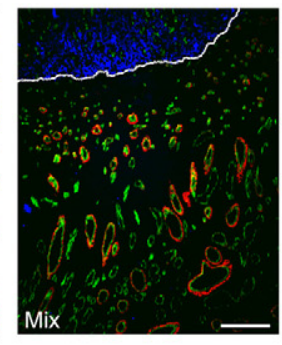

$\mathrm{H}$

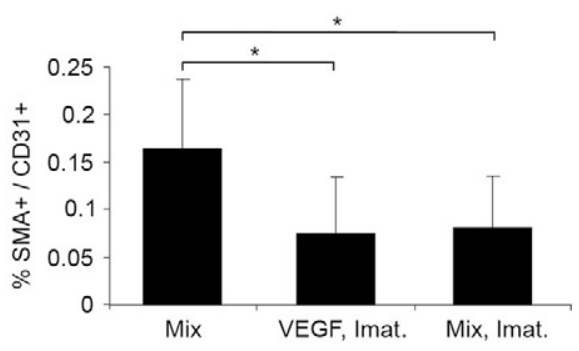

Figure 4. Improved vessel maturation and epithelial morphology in surface transplants coexpressing mVEGF-164 and hPDGF-B and reversion by PDGFR blockade. A-C: Analysis of myofibroblast accumulation and vessel maturation in 2- and 6-week-old transplants of control (Co), mVEGF-164 (VEGF), and mVEGF-164 plus PDGF-B transfectants (Mix) by immunostaining for CD31 (endothelial cells, green), SMA (myofibroblasts and pericytes, red), and nuclei (blue); scale bar, $200 \mu \mathrm{m}$ (Co) and $100 \mu \mathrm{m}$ (VEGF/Mix) D-G: The hPDGF-B-mediated vessel maturation and improved tissue structure are reverted by blockade of PDGFR signaling with imatinib for 3 weeks (week 6 posttransplantation). Staining for SMA (red), CD31 (green), and keratin (blue) in the tumor stroma border (D and $\mathbf{F})$ and tumor tissue (E and $\mathbf{G}$ ) of imatinib-treated (right panels) mVEGF-164 transplants (VEGF, Imat., $\mathbf{D}$ and $\mathbf{E}$ ) and treated mVEGF-164 plus hPDGF-B mixed transplants (Mix, Imat., F and $\mathbf{G}$ ) in comparison with age-matched untreated (left panels) $m V E G F$ and $m V E G F-164$ plus $h P D G F-B$ mixed transplants (Mix); scale bar, $200 \mu \mathrm{m}$ (D and $\mathbf{F})$ and $100 \mu \mathrm{m}(\mathbf{E}$ and $\mathbf{G})$. Note the reduced number of SMA-positive vessels in the upper stroma of mVEGF-164 plus hPDGF-B mixed transplants (Mix, Imat.) and the lack of pericytecovered vessels in the tumor tissue of mVEGF164 plus hPDGF-B mixed transplants (Mix, Imat.) after 3 weeks of treatment with imatinib. Arrowheads mark endothelial free areas in the lacunae in $\mathbf{E}$; arrows show the reduced association of pericytes with the endothelium in $\mathbf{G}$ H: Quantification of SMA-positive area in relation to CD31-stained area demonstrates a significantly increased amount of SMA-coated vessels after 3 weeks of imatinib treatment (week 6 posttransplantation) in untreated mVEGF164 plus hPDGF-B mixed transplants (Mix) compared with treated mVEGF-164 transfectant (VEGF, Imat.) and treated mVEGF-164 plus hPDGF-B mixed transplants (Mix, Imat.). The bars represent the means $\pm \mathrm{SD} ;{ }^{*} P<0.05$.
Invasive Growth of mVEGF-164 and mVEGF-164 plus hPDGF-B-Transfected HaCaT Cells in

\section{Three-Dimensional Organotypic Co-Cultures}

To dissect the effects of both growth factors on tumor growth and invasion in more detail, we analyzed the growth of the respective transfectants in three-dimensional organotypic cultures in vitro, allowing tumor cell growth on an in vivo-like matrix with or without normal human dermal fibroblasts (control, mVEGF-164, hPDGF-B as well as the 1:1 mixture of mVEGF-164 plus hPDGF-B transfectants). Epithelial growth was strongly impaired in all cultures without fibroblasts (Supplemental Figure S3, see http://ajp.amjpathol.org). In co-culture with fibroblasts, mVEGF-164 transfectants alone and the mix- ture of mVEGF-164 plus hPDGF-B transfectants showed invasive growth into the collagen gel starting from week 2, whereas control and hPDGF-B transfectants alone did not invade into the gel (Figure 6A, arrows mark the invasive areas). Similar to our in vivo observations, tumor cell invasion was accompanied by the disruption of basement membrane structures as indicated by the discontinuous collagen IV staining in the co-cultures of the mVEGF-164 transfectants or its almost complete absence in the mVEGF-164 plus hPDGF-B co-cultures (Figure $6 \mathrm{~B}$ arrows, shown for week 3). Thus, these data demonstrate that the invasive in vivo growth behavior that is associated with the expression of mVEGF-164 can be mimicked in the defined three-dimensional organotypic cultures with fibroblasts in vitro. 
A

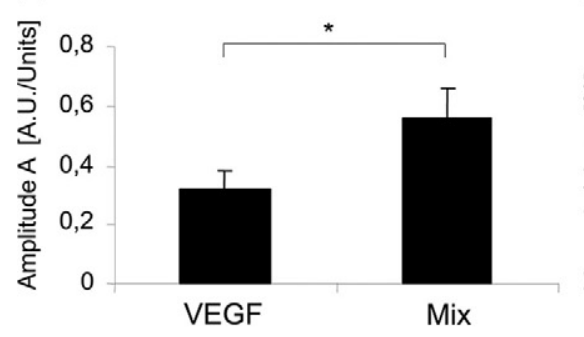

B

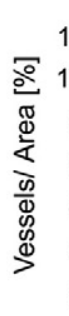

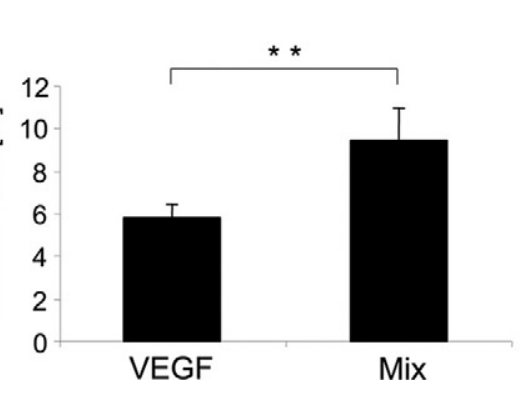

C

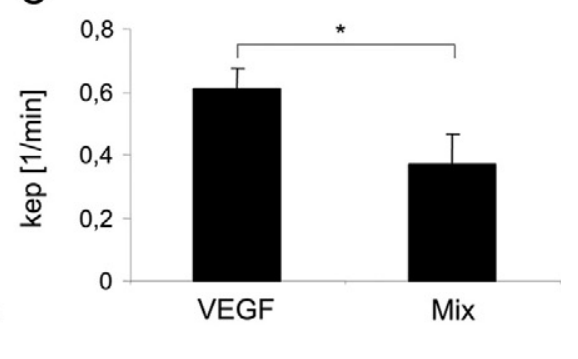

Figure 5. Higher blood volume, vessel density, and lower vessel permeability by the coexpression of mVEGF-164 and hPDGF-B. Values for amplitude (A) and exchange rate constant $\operatorname{kep}(\mathbf{C})$ as calculated from DCE-MRI data and the quantification of vessel density (B) in the vascularized peripheral regions of mVEGF-164 (VEGF) and mVEGF-164 plus hPDGF-B (Mix) transfectant tumors. The amplitude A is a measure of the relative blood volume, and the exchange rate constant kep is highly influenced by blood vessel permeability. The increased amplitude and vessel density in mVEGF-164 plus hPDGF-B mixed tumors as well as the lower kep values indicate improved vascularization and vessel function. The bars represent the means $\pm \mathrm{SD}$, four animals each; ${ }^{*} P<0.05$ and ${ }^{* * *} P<0.001$.

\section{Enhanced MMP Expression in mVEGF-164 Transfectant and mVEGF-164 plus hPDGF-B Mixed Transplants}

We had previously shown that expression of stromal MMP-13 and MMP-9 and of tumor-derived MMP-1 were hallmarks of an invasive tumor stage in highly malignant HaCaT-ras squamous cell carcinomas. ${ }^{42}$ To characterize the tumor stage of the HaCaT mVEGF-164 induced tumors and of the mVEGF-164 plus hPDGF-B mixed tumors in more detail and to pinpoint potential mechanisms behind the invasive growth behavior, we assessed the expression of these invasion associated MMPs in the HaCaT mVEGF-164, mVEGF-164 plus hPDGF-B mixed, and benign hPDGF-B transplants. Indeed, both stromal MMP-9 and MMP-13 were strongly induced in transplants of mVEGF-164 and mVEGF-164 plus hPDGF-B mixed transfectants, thus correlating with their invasive growth behavior and their enhanced angiogenesis. In contrast, only a very faint expression of stromal MMP-9 was detected in the benign HaCaT hPDGF-B transplants, whereas stromal MMP-13 expression was completely absent (Figure 7, A and B). In addition to the stromal expression of MMP-9 and -13, MMP-1 expression was induced in the tumor epithelium of mVEGF-164 transfectants and the mVEGF-164 plus hPDGF-B mixed transplants, whereas again no MMP-1 expression was observed in the benign hPDGF-B transfectant transplants (Figure 7C). Quantification revealed no significant differences in the expression levels of the MMPs in $\mathrm{HaCaT}$ mVEGF-164 transplants versus the mVEGF-164 plus hPDGF-B mixed transplants (Figure 7D). Thus, our data indicate that VEGF is the driving force for the induction of tumor MMP-1 and stromal MMP-9 and MMP-13.

\section{Discussion}

VEGF is a key factor in physiological and pathological angiogenesis, eg, during wound healing, ${ }^{5}$ psoriasis ${ }^{6}$, and cancer. Accordingly, VEGF is overexpressed in many human tumors and correlates with poor prognosis. ${ }^{43-49} \mathrm{How}$ ever, VEGF-induced blood vessels often show increased permeability, leading to hemorrhages and impaired tissue perfusion. ${ }^{18,50}$ To establish mature functional blood vessels, additional growth factors need to cooperate with VEGF. One of the best characterized vessel maturation factors is PDGF-B that promotes the recruitment of PDGFR $\beta$-expressing pericytes to angiogenic vessels. ${ }^{27,51}$ As pericyte-associated blood vessels are refractory to antiangiogenic treatment targeting VEGF, vascular maturation has been proposed as a tumor progression marker. ${ }^{52}$ In the HaCaT skin carcinogenesis model, overexpression of human PDGF-B by nontumorigenic HaCaT cells led to the induction of benign tumor growth. This was mediated by initial stromal activation and angiogenesis induced by fibroblast-derived VEGF-A and hepatocyte growth factor (HGF) that was followed by VEGF down-regulation and subsequent stromal and vascular maturation. ${ }^{28,29}$ To dissect the role of VEGF-A and PDGF-B in the HaCaT skin carcinogenesis model, we stably transfected nontumorigenic $\mathrm{HaCaT}$ cells to express mVEGF-164 and analyzed the in vitro and in vivo growth behavior of the HaCaT transfectants alone as well as the growth of a 1:1 mixture of HaCaT mVEGF-164 and HaCaT hPDGF-B transfectants. Two-dimensional growth of the mVEGF-164 transfectants was not significantly altered, because all analyzed $\mathrm{HaCaT}$ cell lines lacked VEGFR-1 and VEGFR-2 expression, thereby excluding an autocrine effect of VEGF. However, s.c. injection of the HaCaT mVEGF-164 transfectants into nude mice led to formation of malignant, invasive squamous cell carcinomas, demonstrating that the sole expression of mVEGF-164 was sufficient to induce malignant conversion. This revealed a progression promoting effect of VEGF that extended beyond the previously observed VEGF-induced conversion of benign squamous cell carcinoma- 13 keratinocyte tumors to malignant tumor growth. ${ }^{17}$ The HaCaT mVEGF-164-induced tumors appeared red colored and ulcerated, consisting of a heterogeneous tumor mass interrupted by large lacunae. The partial or complete lack of endothelial cell and collagen IV lining indicated a strong impairment in vessel morphology and function, as typically described for VEGF overexpression. ${ }^{53,54}$

To analyze whether additional expression of PDGF-B might improve tissue organization and vessel morphology, we injected a 1:1 mixture of HaCaT mVEGF-164 transfectants and HaCaT hPDGF-B transfectants into nude mice. The resulting tumors reached a similar size as the mVEGF-164 transfectant tumors. However, the tumor 
A
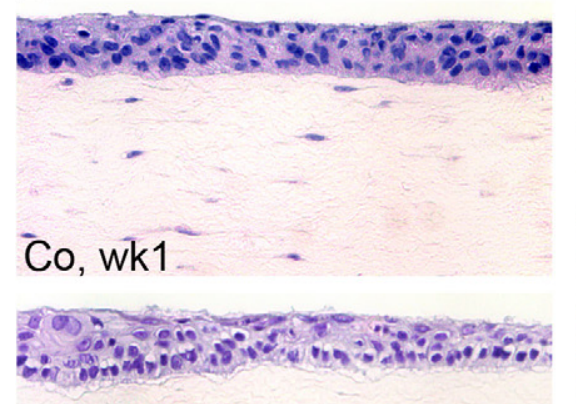

PDGF, wk1

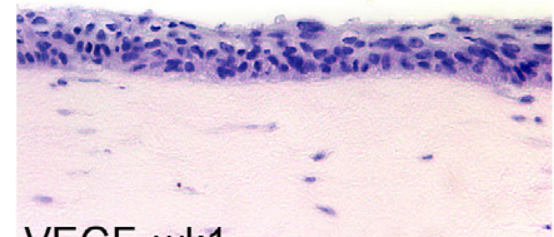

VEGF, wk1

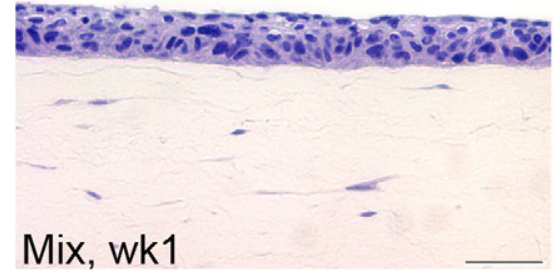

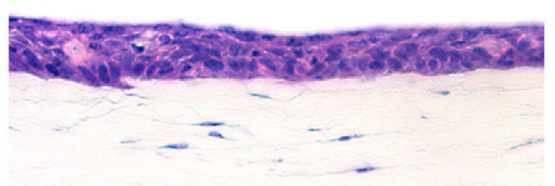

Co, wk2
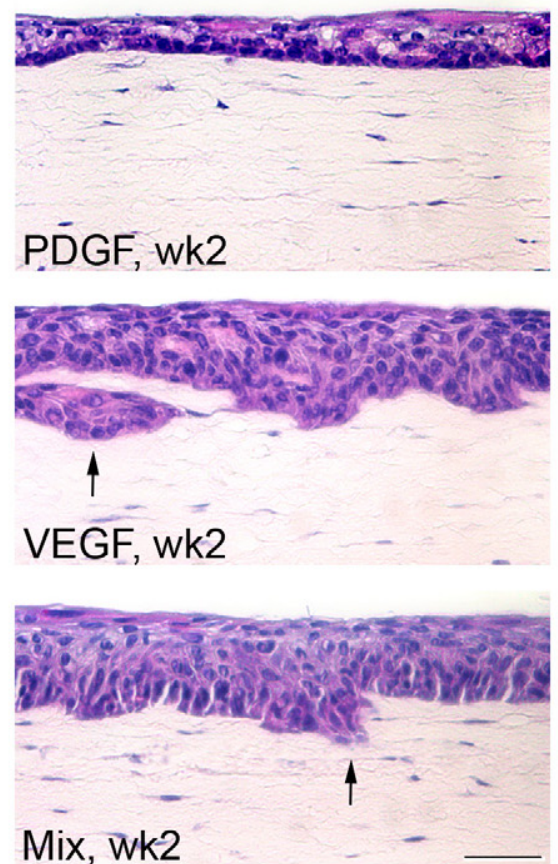
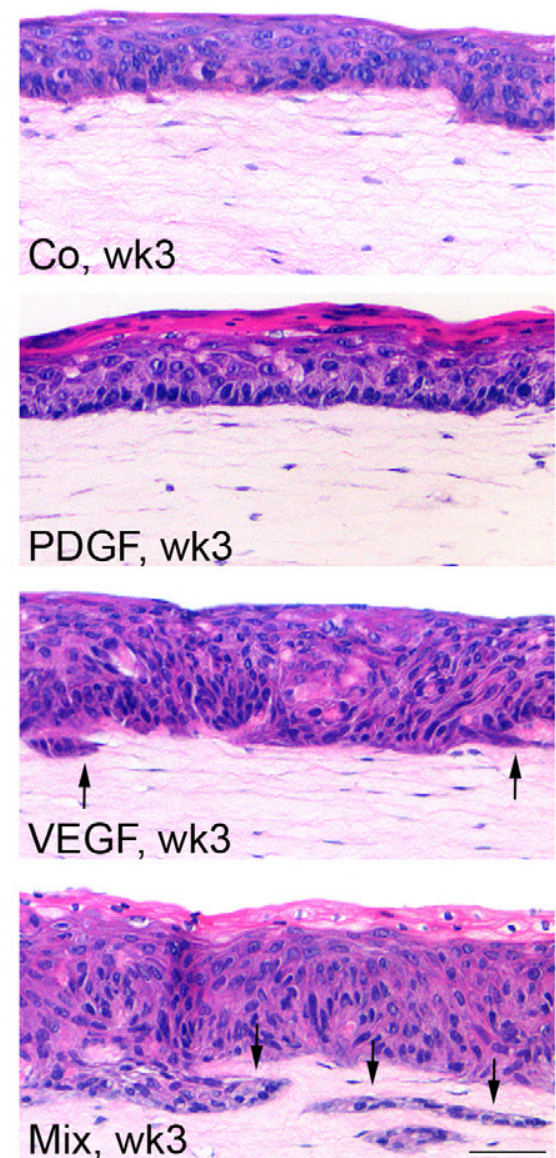

$\mathrm{B}$
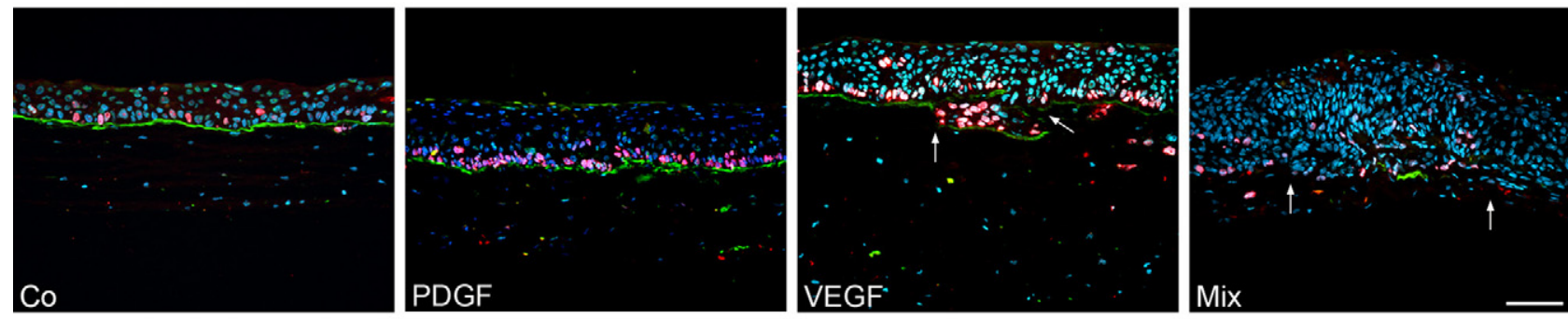

Ki67/ Col IV/ Hoechst

Figure 6. Invasive growth of mVEGF-164 and mVEGF-164 plus hPDGF-B transfectants in organotypic co-cultures with fibroblasts. A: H\&E staining of 1- to 3-week-old organotypic cultures of control transfectants (Co), hPDGF-B transfectants (PDGF), mVEGF-164 transfectants (VEGF), and mVEGF-164 plus hPDGF-B transfectants (Mix) with human dermal fibroblasts. Arrows mark the invasive growth of the tumor cells. Bar, $50 \mu \mathrm{m}$ (all panels). B: Immunofluorescent staining of 3-week-old organotypic co-cultures of control transfectants (Co), hPDGF-B transfectants (PDGF), mVEGF-164 transfectants (VEGF), and mVEGF-164 plus hPDGF-B transfectants (Mix) with fibroblasts against Ki-67 (proliferating cells, red), collagen IV (basement membrane, green), and Hoechst (nuclei, blue); Scale bar, $100 \mu \mathrm{m}$ (all panels). Arrows mark the invasive areas lacking collagen IV staining.

tissue was more compact, being interrupted only by small lacunae. An increase in solid tumor mass and thus in the number of epithelial tumor cells was confirmed by quantifying the area of keratin expressing tumor cells in relation to the total tumor area. In addition to an improved tissue structure, SMA-positive vessels could be detected within the tumor tissue, indicating an improved vessel maturation by the coexpression of PDGF-B when compared with the expression of mVEGF-164 alone.

Kinetic analyses of angiogenesis and epithelial growth in the surface transplants of HaCaT mVEGF-164 trans- fectants demonstrated an earlier and enhanced angiogenesis induction compared with the controls and with benign $\mathrm{HaCaT}$ hPDGF-B transfectants. ${ }^{29}$ However, mVEGF-164 induced the formation of irregular, tortuous blood vessels as already described ${ }^{55-57}$ and of lacunaelike structures in the tumor tissue. Angiogenesis was similarly accelerated and enhanced in the mVEGF-164 plus hPDGF-B mixed surface transplants, but blood vessel growth was more directional and the tumor tissue appeared more compact. Additionally in these mixed transplants, we found an earlier recruitment of SMA-pos- 


\section{A ISH MMP-13}

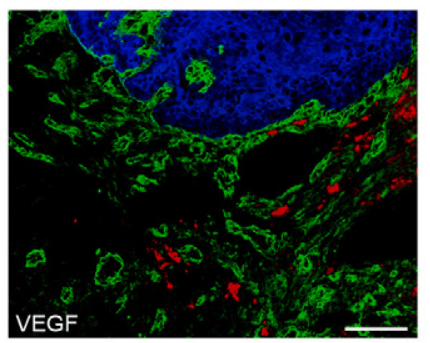

MMP-13/ Col IV/ Keratin

\section{B ISH MMP-9}

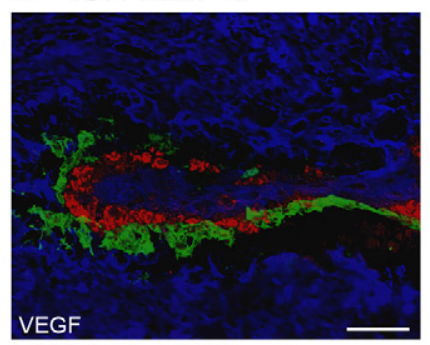

MMP-9/ Col IV/ Keratin

\section{ISH MMP-1}
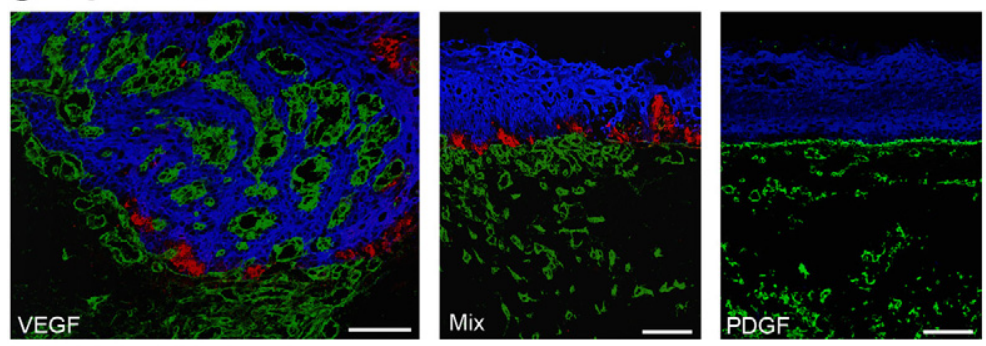

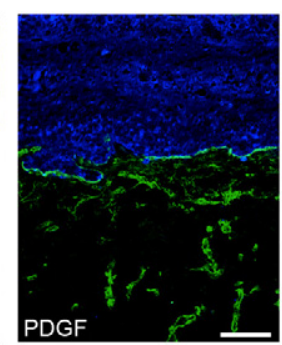

MMP-13/ Col IV/ Keratin
D
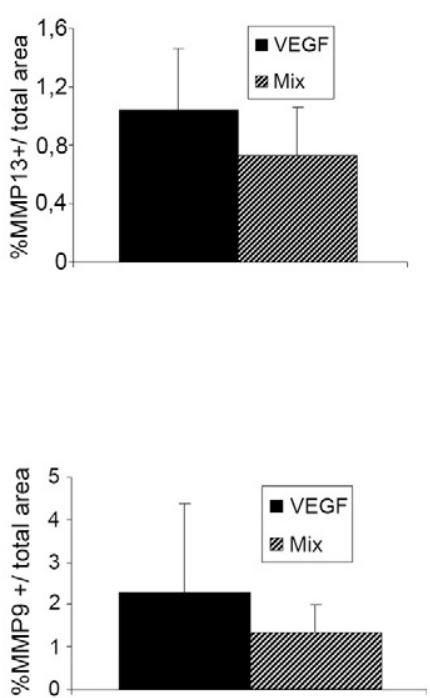

MMP-1/ Col IV/ Keratin

Figure 7. Strong induction of MMPs in mVEGF-164 transfectant and mVEGF-164 plus hPDGF-B mixed surface transplants. A-C: Nonradioactive in situ hybridization with probes specific for mMMP-13 (red, A), mMMP-9 (red, B) and hMMP-1 (red, C), combined with subsequent immunofluorescent staining agains collagen IV (basement membrane, green) and against pan-keratin (tumor cells, blue). A: middle panel, Counterstaining against MMP-9-protein (green) instead of collagen IV is shown. Note the strong induction of stromal MMP-13, MMP-9 and tumor MMP-1 in the mVEGF-164 (VEGF) and mVEGF-164 plus hPDGF-B mixed transplants (Mix); scale bar, $100 \mu \mathrm{m}$ in all panels, except for the left panel in $\mathbf{B}(50 \mu \mathrm{m})$. D: Quantification of the ratio of MMP stained area to the total area demonstrates similar expression levels of MMP-13, -9, and -1 in mVEGF-164 (VEGF) and mVEGF-164 plus hPDGF-B mixed transplants (Mix).

itive cells that were not blood vessel associated and are most probably myofibroblasts. In addition, SMA-positive pericytes were much stronger associated with blood vessels. Since a similar recruitment of SMA-positive cells to the tumor stroma and the tumor blood vessels had been previously observed in surface transplants of HaCaT hPDGF-B transfectants, both effects were most probably mediated by PDGF-B. ${ }^{29}$ Besides enhanced vessel maturation, the epithelial lacunae within the tumor tissue were markedly smaller, and the lacunae were lined by CD31-positive endothelial cells and collagen IV as marker for a vascular basement membrane. This confirmed the results of the s.c. tumors, showing an enhanced vessel maturation and an improved epithelial morphology by the coexpression of hPDGF-B together with mVEGF-164.

To further analyze whether improved vessel maturation and tumor morphology were indeed due to PDGF-B, we blocked PDGFR signaling by treatment with imatinib in surface transplants from week 3 to week 6 posttransplantation. ${ }^{34,35}$ Imatinib reduced the recruitment of myofibro- blasts in mVEGF-164 transfectant transplants, most probably by interfering with signaling of the endogenous, stromal PDGF-B. However, the amount of pericyte-associated blood vessels and the epithelial structure were not altered in the imatinib-treated mVEGF-164 transfectant transplants. In mVEGF-164 plus hPDGF-B mixed transplants that were treated with imatinib for 3 weeks, a significantly decreased amount of SMA-coated blood vessels was observed in the upper stromal part, demonstrating that imatinib efficiently blocked the association of pericytes with newly formed vessels. Even in the tumor tissue, the treatment significantly reduced the association of pericytes with blood vessels, thereby supporting previous studies on the treatment of melanoma with imatinib. ${ }^{58}$ More interestingly, large lacunae with interrupted endothelial cell lining or even devoid of endothelial cells appeared in the tumor tissue after imatinib treatment, resembling the lacunae of the mVEGF-164 transfectant transplants and S.c. tumors. This demonstrated a crucial function of hPDGF-B in vessel maturation and the maintenance of tissue structure. 
We next assessed whether the differences in vessel maturation were reflected by differences in vessel function. Therefore, vessel function and permeability were compared by DCE-MRI of s.c. tumors induced by $\mathrm{HaCaT}$ mVEGF-164 transfectants as well as by the 1:1 mixture of mVEGF-164 plus hPDGF-B transfectants. Postprocessing according to the two compartment model of Brix demonstrated a higher relative blood volume in the mVEGF-164 plus hPDGF-B mixed tumors by the increased amplitude A. Similarly to highly malignant HaCaT-ras A-5RT3 tumors, ${ }^{59}$ the increased amplitude correlated with a higher microvessel density. In contrast, kep, a parameter influenced by the vessel permeability, was higher in the mVEGF-164 transfectant tumors. This suggested the presence of predominantly leaky vessels in mVEGF-164-induced tumors and was in agreement with our histological data showing impaired vessel maturation and the loss of vascular basement membrane structures by the single overexpression of mVEGF-164. Moreover, the increased kep values together with the impaired vessel maturation either in the mVEGF-164 transfectant or in mVEGF-164 plus hPDGF-B mixed tumors treated with imatinib suggest that the large lacunae observed in tumors of both groups might have resulted from nonfunctional, degrading blood vessels. In contrast, PDGF-B obviously leads to a higher vessel density in s.c. tumors by its vessel-stabilizing effects.

Our observations are in clear contrast to previous studies that highlight VEGF as a negative regulator of vessel maturation and pericyte function by suppressing PDGFR signaling, ${ }^{60}$ because we found no negative effect of mVEGF-164 on vessel maturation and function in combination with hPDGF-B in the mVEGF-164 plus hPDGF-B mixed transplants. In accordance with our results, PDGF expression in melanoma and glioma models enhanced tumor growth by stimulating pericyte recruitment, leading to improved vessel function and stability and reduced endothelial cell apoptosis. ${ }^{61,62}$ In glioma, PDGF-B even up-regulated VEGF in the tumor endothelium without affecting pericyte recruitment. ${ }^{62}$ In further support of our observations, combined inhibition of VEGF and PDGF induced a stronger vessel regression than VEGF-inhibition alone, ${ }^{63-65}$ thus improving therapy.

Interestingly, the invasive growth of the mVEGF-164transfected and mVEGF-164 plus hPDGF-B-transfected tumor cells in vivo was not solely mediated by the enhanced angiogenic response, because it could be mimicked in a three-dimensional organotypic co-culture model in vitro that contained as the only stromal component normal human dermal fibroblasts in a collagen type 1 matrix. In this model, epithelial growth was dramatically impaired in cultures without fibroblasts, supporting the essential role of fibroblasts even for the growth of tumorigenic epithelial cells in vitro. ${ }^{66}$ More importantly, in coculture with fibroblasts, mVEGF-164 and mVEGF-164 plus hPDGF-B transfectants showed invasive growth into the collagen gel starting from week 2 , whereas no invasion was observed in co-cultures with the control and the hPDGF-B transfectants. These observations suggest that VEGF plays a predominant role in promoting tumor cell invasion. Invasion was confirmed by the partial or com- plete lack of collagen IV in the basement membrane zone of the invasive epithelia. Thus, three-dimensional co-culture with fibroblasts mimicked the in vivo growth behavior of the respective $\mathrm{HaCaT}$ transfectants, highlighting the crucial role of the tumor microenvironment in supporting tumor invasion. One potential mechanism behind this invasive growth behavior was suggested by analyzing the expression of stromal and tumor-derived MMPs that were previously identified as hallmarks for malignant, invasive HaCaT-ras xenografts. ${ }^{42,67-69}$ We found a similar marked induction of stromal MMP-9 and MMP-13 as well as tumor MMP-1 in transplants of mVEGF-164 transfectant and mVEGF-164 plus hPDGF-B mixed transplants. In contrast, stromal MMP-9 was only marginally expressed in benign HaCaT hPDGF-B transfectant transplants, and stromal MMP-13 and tumor-derived MMP-1 were completely lacking. Since no differences in MMP induction were observed between mVEGF-164 transfectant and mVEGF-164 plus hPDGF-B mixed transplants yet a striking difference was observed when comparing HaCaT hPDGF-B transfectant transplants, we assume that the MMPs were predominantly induced by VEGF. Thus, our results strongly suggest that tumor cell invasion is predominantly mediated by VEGF-A and involves the induced MMPs in tumor and stroma, whereas PDGF-B is responsible for the establishment of a functional, stable neovasculature. However, the detailed mechanisms of MMP induction and the specific contribution of the different stromal cell types (eg, fibroblasts, endothelial cells, inflammatory cells) to tumor invasion and malignancy are matter of further investigations.

In summary, our data demonstrate that the coexpression of VEGF-A together with PDGF-B is necessary to induce the formation of invasive, solid skin squamous cell carcinomas with a stable, functional tumor vasculature. These observations emphasize the importance of multifactorial growth factor inhibition for efficient tumor therapy.

\section{Acknowledgments}

We thank Prof. Dr. Michael Detmar, Institute of Pharmaceutical Sciences, Swiss Federal Institute of Technology (Zurich, Switzerland) for providing the VEGF transfectants. Moreover, we thank Heinrich Steinbauer and Regina Beck for excellent technical assistance.

\section{References}

1. Folkman J: Role of angiogenesis in tumor growth and metastasis Semin Oncol 2002, 29:15-18

2. Folkman J, Klagsbrun M: Angiogenic factors. Science 1987 235:442-447

3. Carmeliet $P$, Jain RK: Angiogenesis in cancer and other diseases Nature 2000, 407:249-257

4. Naumov GN, Akslen LA, Folkman J: Role of angiogenesis in human tumor dormancy: animal models of the angiogenic switch. Cell Cycle 2006, 5:1779-1787

5. Brown LF, Yeo KT, Berse B, Yeo TK, Senger DR, Dvorak HF, van de WL: Expression of vascular permeability factor (vascular endothelial 
growth factor) by epidermal keratinocytes during wound healing. J Exp Med 1992, 176:1375-1379

6. Detmar M, Brown LF, Claffey KP, Yeo KT, Kocher O, Jackman RW, Berse B, Dvorak HF: Overexpression of vascular permeability factor/ vascular endothelial growth factor and its receptors in psoriasis. J Exp Med 1994, 180:1141-1146

7. Ferrara N, Chen H, Davis-Smyth T, Gerber HP, Nguyen TN, Peers D, Chisholm V, Hillan KJ, Schwall RH: Vascular endothelial growth factor is essential for corpus luteum angiogenesis. Nat Med 1998, 4:336-340

8. Neufeld G, Cohen T, Gengrinovitch S, Poltorak Z: Vascular endothelial growth factor (VEGF) and its receptors. FASEB J 1999, 13:9-22

9. Shweiki D, Itin A, Soffer D, Keshet E: Vascular endothelial growth factor induced by hypoxia may mediate hypoxia-initiated angiogenesis. Nature 1992, 359:843-845

10. Grugel S, Finkenzeller G, Weindel K, Barleon B, Marme D: Both $\mathrm{v}$-Ha-Ras and v-Raf stimulate expression of the vascular endothelial growth factor in NIH 3T3 cells. J Biol Chem 1995, 270:25915-25919

11. Rak J, Filmus J, Finkenzeller G, Grugel S, Marme D, Kerbel RS: Oncogenes as inducers of tumor angiogenesis. Cancer Metastasis Rev 1995, 14:263-277

12. Cheng SY, Nagane M, Huang HS, Cavenee WK: Intracerebral tumorassociated hemorrhage caused by overexpression of the vascular endothelial growth factor isoforms VEGF121 and VEGF165 but not VEGF189. Proc Natl Acad Sci USA: 1997, 94:12081-12087

13. Claffey KP, Brown LF, del Aguila LF, Tognazzi K, Yeo KT, Manseau EJ, Dvorak HF: Expression of vascular permeability factor/vascular endothelial growth factor by melanoma cells increases tumor growth, angiogenesis, and experimental metastasis. Cancer Res 1996, $56: 172-181$

14. Oku T, Tjuvajev JG, Miyagawa T, Sasajima T, Joshi A, Joshi R, Finn R, Claffey KP, Blasberg RG: Tumor growth modulation by sense and antisense vascular endothelial growth factor gene expression: effects on angiogenesis, vascular permeability, blood volume, blood flow, fluorodeoxyglucose uptake, and proliferation of human melanoma intracerebral xenografts. Cancer Res 1998, 58:4185-4192

15. Larcher F, Murillas R, Bolontrade M, Conti CJ, Jorcano JL: VEGF/VPF overexpression in skin of transgenic mice induces angiogenesis, vascular hyperpermeability and accelerated tumor development. Oncogene 1998, 17:303-311

16. Smith-McCune K, Zhu YH, Hanahan D, Arbeit J: Cross-species comparison of angiogenesis during the premalignant stages of squamous carcinogenesis in the human cervix and K14-HPV16 transgenic mice. Cancer Res 1997, 57:1294-1300

17. Detmar M, Velasco P, Richard L, Claffey KP, Streit M, Riccardi L, Skobe M, Brown LF: Expression of vascular endothelial growth factor induces an invasive phenotype in human squamous cell carcinomas. Am J Pathol 2000, 156:159-167

18. Cao R, Eriksson A, Kubo H, Alitalo K, Cao Y, Thyberg J: Comparative evaluation of FGF-2-, VEGF-A-, and VEGF-C-induced angiogenesis, lymphangiogenesis, vascular fenestrations, and permeability. Circ Res 2004, 94:664-670

19. Darland DC, D'Amore PA: Blood vessel maturation: vascular development comes of age. J Clin Invest 1999, 103:157-158

20. Benjamin LE, Hemo I, Keshet E: A plasticity window for blood vessel remodelling is defined by pericyte coverage of the preformed endothelial network and is regulated by PDGF-B and VEGF. Development 1998, 125:1591-1598

21. Lindahl P, Johansson BR, Leveen P, Betsholtz C: Pericyte loss and microaneurysm formation in PDGF-B-deficient mice. Science 1997, 277:242-245

22. Hellstrom M, Kalen M, Lindahl P, Abramsson A, Betsholtz C: Role of PDGF-B and PDGFR- $\beta$ in recruitment of vascular smooth muscle cells and pericytes during embryonic blood vessel formation in the mouse. Development 1999, 126:3047-3055

23. Bjarnegard M, Enge M, Norlin J, Gustafsdottir S, Fredriksson S, Abramsson A, Takemoto M, Gustafsson E, Fassler R, Betsholtz C: Endothelium-specific ablation of PDGFB leads to pericyte loss and glomerular, cardiac and placental abnormalities. Development 2004, 131:1847-1857

24. Peres R, Betsholtz C, Westermark B, Heldin CH: Frequent expression of growth factors for mesenchymal cells in human mammary carcinoma cell lines. Cancer Res 1987, 47:3425-3429

25. Bronzert DA, Pantazis P, Antoniades HN, Kasid A, Davidson N,
Dickson RB, Lippman ME: Synthesis and secretion of platelet-derived growth factor by human breast cancer cell lines. Proc Natl Acad Sci USA 1987, 84:5763-5767

26. Abramsson A, Berlin O, Papayan H, Paulin D, Shani M, Betsholtz C: Analysis of mural cell recruitment to tumor vessels. Circulation 2002, 105:112-117

27. Abramsson A, Lindblom P, Betsholtz C: Endothelial and nonendothelial sources of PDGF-B regulate pericyte recruitment and influence vascular pattern formation in tumors. J Clin Invest 2003, 112: $1142-1151$

28. Skobe M, Fusenig NE: Tumorigenic conversion of immortal human keratinocytes through stromal cell activation. Proc Natl Acad Sci USA 1998, 95:1050-1055

29. Lederle W, Stark HJ, Skobe M, Fusenig NE, Mueller MM: Plateletderived growth factor-BB controls epithelial tumor phenotype by differential growth factor regulation in stromal cells. Am J Pathol 2006, 169:1767-1783

30. Boukamp P, Petrussevska RT, Breitkreutz D, Hornung J, Markham A, Fusenig NE: Normal keratinization in a spontaneously immortalized aneuploid human keratinocyte cell line. J Cell Biol 1988, 106:761-771

31. Stacey A, Doyle A: Routine testing of cell cultures and their products for mycoplasma contamination. Methods Mol Biol 1997, 75:305-311

32. Mueller MM, Fusenig NE: Tumor-stroma interactions directing phenotype and progression of epithelial skin tumor cells. Differentiation 2002, 70:486-497

33. Mueller MM, Fusenig NE: Friends or foes-bipolar effects of the tumour stroma in cancer. Nat Rev Cancer 2004, 4:839-849

34. Druker BJ: STI571 (Gleevec) as a paradigm for cancer therapy. Trends Mol Med 2002, 8:S14-S18

35. Kubo T, Piperdi S, Rosenblum J, Antonescu CR, Chen W, Kim HS, Huvos AG, Sowers R, Meyers PA, Healey JH, Gorlick R: Plateletderived growth factor receptor as a prognostic marker and a therapeutic target for imatinib mesylate therapy in osteosarcoma. Cancer 2008, 112:2119-2129

36. Boukamp P, Stanbridge EJ, Foo DY, Cerutti PA, Fusenig NE: c-Ha-ras oncogene expression in immortalized human keratinocytes (HaCaT) alters growth potential in vivo but lacks correlation with malignancy. Cancer Res 1990, 50:2840-2847

37. Moorman AF, De Boer PA, Vermeulen JL, Lamers WH: Practical aspects of radio-isotopic in situ hybridization on RNA. Histochem $J$ 1993, 25:251-266

38. Brix G, Semmler W, Port R, Schad LR, Layer G, Lorenz WJ: Pharmacokinetic parameters in CNS Gd-DTPA enhanced MR imaging. J Comput Assist Tomogr 1991, 15:621-628

39. Charvat S, Duchesne M, Parvaz P, Chignol MC, Schmitt D, Serres M The up-regulation of vascular endothelial growth factor in mutated $\mathrm{Ha}$-ras $\mathrm{HaCaT}$ cell lines is reduced by a farnesyl transferase inhibitor. Anticancer Res 1999, 19:557-561

40. Hellstrom M, Gerhardt H, Kalen M, Li X, Eriksson U, Wolburg H, Betsholtz C: Lack of pericytes leads to endothelial hyperplasia and abnormal vascular morphogenesis. J Cell Biol 2001, 153:543-553

41. Lindblom P, Gerhardt H, Liebner S, Abramsson A, Enge M, Hellstrom M, Backstrom G, Fredriksson S, Landegren U, Nystrom HC, Bergstrom G, Dejana E, Ostman A, Lindahl P, Betsholtz C: Endothelial PDGF-B retention is required for proper investment of pericytes in the microvessel wall. Genes Dev 2003, 17:1835-1840

42. Vosseler S, Lederle W, Airola K, Obermueller E, Fusenig NE, Mueller MM: Distinct progression-associated expression of tumor and stromal MMPs in $\mathrm{HaCaT}$ skin SCCs correlates with onset of invasion. Int $\mathrm{J}$ Cancer 2009, 125:2296-2306

43. Zhang J, Jia Z, Li Q, Wang L, Rashid A, Zhu Z, Evans DB, Vauthey JN, Xie K, Yao JC: Elevated expression of vascular endothelial growth factor correlates with increased angiogenesis and decreased progression-free survival among patients with low-grade neuroendocrine tumors. Cancer 2007, 109:1478-1486

44. Gratzinger D, Zhao S, Tibshirani RJ, Hsi ED, Hans CP, Pohlman B, Bast M, Avigdor A, Schiby G, Nagler A, Byrne GE Jr, Lossos IS, Natkunam Y: Prognostic significance of VEGF. VEGF receptors, and microvessel density in diffuse large B cell lymphoma treated with anthracycline-based chemotherapy. Lab Invest 2008, 88:38-47

45. Han H, Silverman JF, Santucci TS, Macherey RS, d'Amato TA, Tung MY, Weyant RJ, Landreneau RJ: Vascular endothelial growth factor expression in stage I non-small cell lung cancer correlates with 
neoangiogenesis and a poor prognosis. Ann Surg Oncol 2001, 8:72-79

46. Kamat AA, Merritt WM, Coffey D, Lin YG, Patel PR, Broaddus R, Nugent E, Han LY, Landen CN Jr, Spannuth WA, Lu C, Coleman RL, Gershenson DM, Sood AK: Clinical and biological significance of vascular endothelial growth factor in endometrial cancer. Clin Cancer Res 2007, 13:7487-7495

47. Ellis LM: Preclinical data targeting vascular endothelial growth factor in colorectal cancer. Clin Colorectal Cancer 2004, 4(Suppl 2): S55-S61

48. Smith BD, Smith GL, Carter D, Sasaki CT, Haffty BG: Prognostic significance of vascular endothelial growth factor protein levels in oral and oropharyngeal squamous cell carcinoma. J Clin Oncol 2000, 18:2046-2052

49. Gasparini G: Prognostic value of vascular endothelial growth factor in breast cancer. Oncologist 2000, 5(Suppl 1):37-44

50. Nagy JA, Benjamin L, Zeng H, Dvorak AM, Dvorak HF: Vascular permeability, vascular hyperpermeability and angiogenesis. Angiogenesis 2008, 11:109-119

51. Gerhardt H, Betsholtz C: Endothelial-pericyte interactions in angiogenesis. Cell Tissue Res 2003, 314:15-23

52. Eberhard A, Kahlert S, Goede V, Hemmerlein B, Plate KH, Augustin HG: Heterogeneity of angiogenesis and blood vessel maturation in human tumors: implications for antiangiogenic tumor therapies. Cancer Res 2000, 60:1388-1393

53. Ferrara N: VEGF as a therapeutic target in cancer. Oncology 2005, 69(Suppl 3):11-16

54. Carmeliet P: VEGF as a key mediator of angiogenesis in cancer. Oncology 2005, 69(Suppl 3):4-10

55. Detmar M, Brown LF, Schon MP, Elicker BM, Velasco P, Richard L, Fukumura D, Monsky W, Claffey KP, Jain RK: Increased microvascular density and enhanced leukocyte rolling and adhesion in the skin of VEGF transgenic mice. J Invest Dermatol 1998, 111:1-6

56. Xia YP, Li B, Hylton D, Detmar M, Yancopoulos GD, Rudge JS: Transgenic delivery of VEGF to mouse skin leads to an inflammatory condition resembling human psoriasis. Blood 2003, 102:161-168

57. Kunstfeld R, Hirakawa S, Hong YK, Schacht V, Lange-Asschenfeldt B, Velasco P, Lin C, Fiebiger E, Wei X, Wu Y, Hicklin D, Bohlen P, Detmar M: Induction of cutaneous delayed-type hypersensitivity reactions in VEGF-A transgenic mice results in chronic skin inflammation associated with persistent lymphatic hyperplasia. Blood 2004, 104:1048-1057

58. Hasumi Y, Klosowska-Wardega A, Furuhashi M, Ostman A, Heldin $\mathrm{CH}$, Hellberg C: Identification of a subset of pericytes that respond to combination therapy targeting PDGF and VEGF signaling. Int J Cancer 2007, 121:2606-2614

59. Kiessling F, Krix M, Heilmann M, Vosseler S, Lichy M, Fink C, Farhan N, Kleinschmidt K, Schad L, Fusenig NE, Delorme S: Comparing dynamic parameters of tumor vascularization in nude mice revealed by magnetic resonance imaging and contrast-enhanced intermittent power Doppler sonography. Invest Radiol 2003, 38:516-524

60. Greenberg JI, Shields DJ, Barillas SG, Acevedo LM, Murphy E, Huang J, Scheppke L, Stockmann C, Johnson RS, Angle N, Cheresh DA: A role for VEGF as a negative regulator of pericyte function and vessel maturation. Nature 2008, 456:809-813

61. Furuhashi M, Sjoblom T, Abramsson A, Ellingsen J, Micke P, Li H, Bergsten-Folestad E, Eriksson U, Heuchel R, Betsholtz C, Heldin CH, Ostman A: Platelet-derived growth factor production by B16 melanoma cells leads to increased pericyte abundance in tumors and an associated increase in tumor growth rate. Cancer Res 2004, 64:2725-2733

62. Guo P, Hu B, Gu W, Xu L, Wang D, Huang HJ, Cavenee WK, Cheng SY: Platelet-derived growth factor-B enhances glioma angiogenesis by stimulating vascular endothelial growth factor expression in tumor endothelia and by promoting pericyte recruitment. Am J Pathol 2003, 162:1083-1093

63. Bergers G, Song S, Meyer-Morse N, Bergsland E, Hanahan D: Benefits of targeting both pericytes and endothelial cells in the tumor vasculature with kinase inhibitors. J Clin Invest 2003, 111:1287-1295

64. Erber R, Thurnher A, Katsen AD, Groth G, Kerger H, Hammes HP. Menger MD, Ullrich A, Vajkoczy P: Combined inhibition of VEGF and PDGF signaling enforces tumor vessel regression by interfering with pericyte-mediated endothelial cell survival mechanisms. FASEB $J$ 2004, 18:338-340

65. Sennino B, Kuhnert F, Tabruyn SP, Mancuso MR, Hu-Lowe DD, Kuo CJ, McDonald DM: Cellular source and amount of vascular endothelial growth factor and platelet-derived growth factor in tumors determine response to angiogenesis inhibitors. Cancer Res 2009, 69: 4527-4536

66. Szabowski A, Maas-Szabowski N, Andrecht S, Kolbus A, SchorppKistner M, Fusenig NE, Angel P: C-Jun and JunB antagonistically control cytokine-regulated mesenchymal-epidermal interaction in skin. Cell 2000, 103:745-755

67. Vosseler S, Mirancea N, Bohlen P, Mueller MM, Fusenig NE: Angiogenesis inhibition by vascular endothelial growth factor receptor-2 blockade reduces stromal matrix metalloproteinase expression, normalizes stromal tissue, and reverts epithelial tumor phenotype in surface heterotransplants. Cancer Res 2005, 65:1294-1305

68. Miller DW, Vosseler S, Mirancea N, Hicklin DJ, Bohlen P, Volcker HE, Holz FG, Fusenig NE: Rapid vessel regression, protease inhibition, and stromal normalization upon short-term vascular endothelial growth factor receptor 2 inhibition in skin carcinoma heterotransplants. Am J Pathol 2005, 167:1389-1403

69. Airola K, Fusenig NE: Differential stromal regulation of MMP-1 expression in benign and malignant keratinocytes. J Invest Dermatol 2001, 116:85-92 